\title{
INTERVENÇÃO EDUCATIVA SOBRE USO DE MÍDIAS DIGITAIS NA PRIMEIRA INFÂNCIA
}

\author{
Maíra Lopes Almeida \\ Laura Canani da Rosa \\ Gabriela Vescovi \\ Bruna Gabriella Pedrotti \\ Manoela Yustas Mallmann \\ Giana Bitencourt Frizzo \\ Universidade Federal do Rio Grande do Sul, Porto Alegre-RS, Brasil
}

\begin{abstract}
RESUMO
O uso das mídias digitais por bebês é um fenômeno recente. Algumas evidências apontam para associações do uso de telas na primeira infância e desfechos adversos no desenvolvimento. O objetivo deste estudo é apresentar o processo de construção, implementação e avaliação do estudo-piloto de uma intervenção educativa para pais, profissionais da saúde e educadores sobre uso de mídias digitais na primeira infância. A intervenção segue o modelo de educação continuada, consistiu em quatro encontros presenciais no formato expositivo seguido de discussão em grupo e foi avaliada em termos da aprendizagem dos participantes e satisfação com o curso. Participaram 16 pessoas, majoritariamente profissionais de saúde e educação. Os resultados indicaram melhora significativa nos conhecimentos do tema e satisfação com a intervenção, sendo discutidos de forma a subsidiar o desenvolvimento de novas intervenções sobre a temática.
\end{abstract}

Palavras-chave: Mídias digitais; Criança; Intervenção precoce.

\section{EDUCATIONAL INTERVENTION ON THE USE OF DIGITAL MEDIA IN EARLY CHILDHOOD}

\section{Abstract}

The use of digital media by babies is a recent phenomenon. Some evidence highlights associations between the use of screens in early childhood and adverse developmental outcomes. This study aims to report the development, implementation, and evaluation processes of an educational intervention for parents, health professionals, and educators on the use of digital media in early childhood. The intervention follows the model of continuing education, with four face-to-face meetings and presentations followed by group discussions. It was evaluated by participants' learning and satisfaction with the course. Sixteen people participated, mostly health and education professionals. The results indicate a significant improvement in participants' knowledge and high satisfaction rates. Findings are discussed to support the development of new interventions on the subject.

Keywords: Technology; Children; Early intervention. 


\section{INTERVENCIÓN EDUCATIVA SOBRE EL USO DE MEDIOS DIGITALES EN LA PRIMERA INFANCIA}

\section{RESUMEN}

El uso de medios digitales por parte de los bebés es un fenómeno reciente. Algunas evidencias apuntan asociaciones entre el uso de pantallas en la primera infancia y los resultados adversos del desarrollo. El objetivo del presente estudio es presentar el proceso de construcción, implementación y evaluación del estudio piloto de una intervención educativa para padres, profesionales de la salud y educadores sobre el uso de medios digitales en la primera infancia. La intervención sigue el modelo de educación continua y consistió en cuatro reuniones presenciales en formato expositivo, seguidas de discusión grupal y aún fue evaluada en términos del aprendizaje y satisfacción de los participantes con el curso. Participaron 16 personas, en su mayoría identificadas como profesionales de la salud y de la educación. Los resultados indicaron una mejora significativa en el conocimiento del tema y satisfacción con la intervención, siendo discutida con el fin de apoyar el desarrollo de nuevas intervenciones sobre el tema.

Palabras clave: Medios digitales; Niños; Intervenciónes tempranas.

O uso de mídias digitais, compreendidas como conjunto de dispositivos, formatos e métodos de comunicação que veiculam conteúdo por meio de sinais digitais como na internet, televisão e em redes de computadores e telefonia (American Psychological Association, 2019), é um fenômeno recente e comum no cotidiano familiar. Dispositivos móveis, como tablets e smartphones, tornaram-se parte da rotina, mantendo as pessoas constantemente conectadas.

O levantamento norte americano Common Sense Media, realizado em 2020, mostrou que esse uso tende a variar nos primeiros anos de vida, de modo que se nota, em média, um acréscimo substancial dos primeiros 24 meses de vida (49 minutos) para os 25 aos 48 meses (2 horas e 30 minutos) (Rideout \& Robb, 2020). Além disso, constatou-se que a maioria dos pais e mães não costumam relatar preocupações quanto à quantidade de tempo de tela, à qualidade do conteúdo acessado e ao impacto desse uso para o desenvolvimento infantil.

Esse mesmo levantamento indicou que $97 \%$ das famílias com crianças de até oito anos possuíam ao menos um celular, enquanto o uso de tablets chegou a $75 \%$ (Rideout \& Robb, 2020). Ainda, observa-se que as crianças são cada vez mais cedo introduzidas às mídias digitais, com dados apontando que bebês de quatro meses já fazem algum uso desses dispositivos (American Academy of Pediatrics [AAP], 2016; Kabali et al., 2015).

Os números destacam o frequente uso das mídias digitais nas famílias e dos recursos tecnológicos pelos bebês (Brito et al., 2017). No entanto, as sociedades de pediatria recomendam que crianças de até dois anos não sejam expostas às telas e, caso o façam, a partir dos 18 meses, é indicado que o uso ocorra na presença de um cuidador (AAP, 2016). As diretrizes baseiam-se principalmente na falta de consenso sobre os riscos e benefícios das telas para o desenvolvimento infantil. 
Os riscos apontados na literatura dizem respeito a prejuízos na capacidade de autorregulação, no desenvolvimento da linguagem, cognição e atenção (Radesky et al., 2014), bem como dificuldades de comportamento (McDaniel \& Radesky, 2018b). O uso intenso de mídias por crianças também está associado à obesidade, ao sedentarismo (AAP, 2016; (Domingues-Montanari, 2017) e a problemas de sono (Cheung et al., 2017). Já os benefícios estão relacionados à possibilidade de aproximar familiares distantes por meio de chamadas de vídeo, proporcionar momentos de lazer e entretenimento à família, bem como uma oportunidade de trocas e aprendizados para o bebê, principalmente após os dois anos e com o auxílio de um cuidador que lhe auxilie a dar sentido ao conteúdo assistido (AAP, 2016; Coyne et al., 2017).

Cabe ressaltar que os padrões da utilização de mídia digital são estabelecidos na primeira infância, o que sublinha a necessidade de prevenção e intervenção precoce a fim de criar rotinas mais saudáveis nesse momento do desenvolvimento (McArthur et al., 2020). No cenário internacional, essas intervenções têm se tornado mais comuns (Wu et al., 2016).

No entanto, no Brasil, a revisão de literatura apontou escassez de pesquisas na área, de modo que nenhuma intervenção foi encontrada (Frizzo et al., 2017). De forma geral, estudos que descrevam o passo-a-passo de como desenvolver uma intervenção, principalmente de forma sistemática, são raros na literatura. Sendo assim, os estudos prévios conduzidos pelo grupo de pesquisa colaboraram para nortear aspectos importantes para o planejamento de uma intervenção específica sobre o uso de mídias digitais para o contexto brasileiro (Frizzo et al., 2017).

Em 2018, foi iniciado um projeto de divulgação científica. Inicialmente, foi realizada a produção de uma vídeo-série intitulada "Bebês \& Tecnologias", disponível no YouTube. A vídeo-série conta com 11 episódios e apresentou retornos positivos do público em geral, considerando comentários, número de visualizações e de curtidas. Nesse contexto, iniciou-se o processo de desenvolvimento do estudo-piloto da intervenção que será abordado neste estudo.

De acordo com o cenário contemporâneo do uso de mídias digitais na primeira infância, pais, cuidadores, profissionais de saúde e educadores podem apresentar questionamentos sobre como utilizar estes dispositivos da melhor forma possível, minimizando riscos e maximizando benefícios. O presente estudo adotou a premissa de que a intervenção educativa é um meio eficaz para transmitir conhecimento teóricoprático sobre o uso de mídias digitais na primeira infância (Pedrotti, 2019). Destaca-se que intervenções educativas realizadas com o mesmo objetivo de transmitir e aprimorar conhecimentos, ainda que sobre outros tópicos centrais, demonstraram eficácia e efetividade em outros contextos (Gholamian et al., 2019; Harrison et al., 2006; Lewis, 2020; Machado et al., 2010; Silva et al., 2018; Torquato et al., 2019).

Diante disso, o objetivo deste estudo é apresentar o processo de construção, implementação e avaliação do estudo-piloto de uma intervenção educativa para pais, profissionais da saúde e educadores sobre uso de mídias digitais na primeira infância. Pretende-se avaliar esse estudo-piloto a partir dos conhecimentos adquiridos pelos participantes acerca da temática, bem como a satisfação deles com a intervenção. 


\section{ELABORAÇÃo dA INTERVENÇÃo}

O primeiro passo para desenvolver a intervenção referiu-se à escolha dos temas que seriam abordados e de que forma. Nesse momento do planejamento, o grupo de pesquisa contava com cinco doutorandas, uma mestranda e uma bolsista da graduação. Houve uma reunião entre todas as integrantes com a professora coordenadora do grupo para a definição e elaboração da intervenção. Uma doutoranda ficou responsável pelo planejamento junto com uma graduanda em Psicologia, bolsista de divulgação científica.

De acordo com o objetivo principal, esperava-se realizar uma intervenção educativa com a população de forma sistematizada, isto é, compilar as evidências sobre o uso de mídias digitais por bebês em um modelo de aprendizagem baseado na continuidade. Por isso, optou-se por um formato de palestras com quatro encontros.

Após a escolha do formato, foram definidos os temas a serem trabalhados. $\mathrm{O}$ critério utilizado foi a análise das visualizações e do engajamento dos vídeos da série "Bebês \& Tecnologias" no canal do Youtube do grupo. Assim, selecionou-se quatro temas com maior número de visualizações e de curtidas: "Como fazer um bom uso de tecnologias com o bebê"; "Relação cuidadores-criança e as tecnologias"; "A influência das telas no sono" e; "A influência das telas na aprendizagem".

Ainda na etapa do planejamento, destacam-se alguns passos anteriores à execução: a seleção de instrumentos para avaliação da intervenção e uma pré-aplicação da intervenção com pequeno grupo (Runfola et al., 2014). Além das discussões teóricas que subsidiaram toda a elaboração, essas etapas são cruciais para intervenções e não podem ser negligenciadas, uma vez que guiam a compreensão do que será realizado e propiciam adaptações antes do início (Robson \& McCartan, 2016).

Para a escolha dos instrumentos, foi necessário definir quais aspectos seriam relevantes para considerar que os objetivos da intervenção foram cumpridos. Na discussão para essa definição, concluiu-se que os aspectos a serem avaliados diziam respeito ao conhecimento que o indivíduo adquiriu devido à participação na intervenção e à satisfação com o curso. Assim, o segundo passo foi realizar uma revisão da literatura científica sobre questionários que avaliassem esses pontos.

No caso do primeiro aspecto, não foram encontrados estudos que tivessem abordado o tema no mesmo formato ou com tópicos semelhantes (Schmidt et al., 2012; Wu et al., 2016). Assim, a partir de revisão de literatura e do conhecimento adquirido sobre a temática nas pesquisas anteriores do grupo, criou-se um questionário a ser aplicado antes do início das palestras e reaplicado ao final dos encontros. É importante ressaltar que esse questionário foi construído baseado na literatura científica sobre o tema, discutido pelas pós-graduandas no grupo de pesquisa e revisado por experts que não participaram da criação inicial (a professora coordenadora e uma pós-doutoranda especialista na temática).

Esse instrumento intitulado "Questionário de conhecimentos sobre o uso de mídias digitais por bebês" é composto por 12 itens que contêm perguntas para serem respondidas com "sim" ou "não". As perguntas contemplam tópicos abordados nas palestras, como por exemplo, "a exposição de bebês à TV está associada com problemas na qualidade e na rotina do sono?", "existem recomendações oficiais de sociedades de pediatria para o uso de tecnologias por bebês?" e outros. Ao final, constava uma pergunta aberta: "Algo mais que você gostaria de acrescentar?".

Já sobre a satisfação com o curso, a revisão apontou para um questionário utilizado em cursos de educação continuada com formato parecido com a intervenção 
educativa. Essa escala é baseada no modelo de Kirkpatrick e Kirkpatrick (2016), que define a satisfação como o primeiro de quatro estágios consecutivos para a avaliação da educação continuada: "reação", "aprendizagem", "comportamento" e "resultados". A satisfação é sinônimo de "reação" e é um construto importante do contexto de aprendizagem, que permite a transferência de habilidades, conhecimentos e competências. Nesse modelo, a satisfação é considerada como uma atitude e é avaliada em três níveis: afetivo, cognitivo e comportamental (Kirkpatrick \& Kirkpatrick, 2016).

De acordo com esse referencial teórico, o questionário se intitula "Affective Behavioral - Cognitive - Satisfaction Questionnaire" [Questionário de Satisfação Afetivo-Comportamental-Cognitivo], foi desenvolvido em inglês e é composto por 11 itens dispostos em uma escala likert de 5 pontos (Meng et al., 2018). Ao final, as pontuações são somadas e maiores escores indicam maior satisfação. A escala se divide em três subescalas: afetiva, comportamental e cognitiva. Alguns exemplos dos tópicos abordados nos itens são: a) afetivo (3 itens - 15) - o tom emocional positivo durante as palestras e a motivação do palestrante; b) comportamental ( 2 itens) - a disponibilidade do material utilizado na palestra e a preparação do palestrante para o encontro e; c) cognitivo (6 itens) - se o conhecimento foi passado de forma clara e se considera que foi adquirido conhecimento. Foram apresentadas propriedades psicométricas adequadas, como evidências de validade de face, de constructo, que indicou a existência de três fatores, e consistência interna das subescalas avaliada por meio do alpha de Cronbach que variou de 0.60 a 0.83 .

Ao identificar que a escala seria útil para colaborar com a intervenção, seguiramse os passos preconizados para adaptação transcultural de instrumentos (Borsa et al., 2012). Inicialmente, foi feito contato com os autores responsáveis pela escala (Meng et al., 2018), que autorizaram a tradução, adaptação e validação da escala pelo grupo de pesquisa. Após obter essa autorização, duas responsáveis pela intervenção realizaram uma primeira tradução da escala, que depois foi avaliada por todas as outras pósgraduandas.

A partir disso, obteve-se por meio de consenso uma síntese que, então, passou por nova avaliação. Essa versão foi avaliada pela coordenadora do grupo de pesquisa, que não tinha tido contato com a escala original e as traduções até aquele momento. Cabe esclarecer que os outros passos relativos à adaptação transcultural e validação da escala ainda estão em andamento. Definida a versão final dos instrumentos, procedeu-se ao outro passo fundamental do planejamento, a pré-aplicação da intervenção com pequeno grupo a fim de verificar possíveis problemas e sugestões para a implementação.

\section{DIVULGAÇÃO DA INTERVENÇÃO E PRÉ-APLICAÇÃO DA INTERVENÇÃO EM PEQUENO GRUPO}

Concomitante à seleção dos instrumentos, aconteceu a divulgação da intervenção e a pré-aplicação. As redes sociais do grupo, da universidade e publicação no jornal local foram os meios escolhidos para a divulgação que ocorreu em setembro de 2019, cerca de um mês antes do início do curso. Divulgou-se algumas imagens com as principais informações da intervenção, como data, hora, local, temas abordados e instruções para as inscrições.

Além disso, criou-se um evento no Facebook para tal. Imediatamente, chamou a atenção do grupo o interesse da população pelo curso. Outros meios de comunicação, como sites de notícias e um jornal local, convidaram as pesquisadoras para reportagens sobre a temática e logo todas as vagas estavam preenchidas. Em duas semanas, foram 
obtidas mais de duzentas inscrições.

Durante esse processo de divulgação, desenvolveu-se um estudo prévio da intervenção com um pequeno grupo na Creche da universidade. Esse evento ocorreu um mês antes do início da intervenção e contou com 9 participantes, sendo 3 cuidadores de crianças que frequentavam a creche e 6 professoras. $O$ encontro foi elaborado de maneira a abordar brevemente todos os quatro conteúdos. Também foi possível testar a plataforma digital interativa Mentimeter, na qual os participantes colocavam suas dúvidas anonimamente.

Ao final, foi entregue um questionário aberto e respondido anonimamente sobre quais melhorias os participantes sugeriam para o encontro e os conteúdos. As respostas foram todas positivas, com agradecimentos às pesquisadoras e indicando maior interesse em continuar debatendo o assunto. Assim, ao finalizar a escolha, criação e adaptação dos instrumentos, divulgação do curso e pré-aplicação da intervenção, iniciou-se a implementação da intervenção que será detalhada a seguir. Destaca-se que este estudo se baseia na resolução 510/2016, segundo a qual "pesquisa realizada com base de dados, cujas informações são agregadas, sem possibilidade de identificação individual", dispensa o registro e avaliação por Comitê de Ética" (Conselho Nacional de Saúde, 2016).

\section{IMPLEMENTAÇÃO DA INTERVENÇÃO}

A implementação da intervenção foi realizada em quatro encontros semanais, nas quartas-feiras de outubro do ano de 2019, tendo início no dia 9 e término no dia 30. Os encontros foram presenciais e ocorreram nas dependências da universidade, com uma duração média de uma hora. Cada encontro foi coordenado por uma pós-graduanda do grupo de pesquisa e uma graduanda, responsável pela produção dos vídeos do grupo no Youtube.

Nessa implementação, destaca-se que, inicialmente, a principal dificuldade encontrada foi o alto número de inscritos. Com a ampla divulgação, mais de 200 pessoas se inscreveram, excedendo o planejamento inicial que esperava em torno de 25 pessoas. Para solucionar esse problema, foram enviados e-mails aos interessados a fim de confirmar a participação. Como o número de respostas de confirmação obtido foi próximo ao número esperado, manteve-se o planejamento inicial no que diz respeito às questões operacionais, como sala e impressão de instrumentos. Os encontros seguiram conforme o seguinte protocolo (Tabela 1). 


\section{Tabela 1}

Descrição do protocolo da intervenção educativa

\begin{tabular}{|c|c|c|c|}
\hline Encontro & Objetivo & Atividade & Orientação \\
\hline $\begin{array}{l}\text { Primeiro encontro: } \\
\text { Como fazer um bom } \\
\text { uso das mídias } \\
\text { digitais } \\
\begin{array}{l}\text { Tempo previsto: } 40 \\
\text { minutos }\end{array}\end{array}$ & $\begin{array}{l}\text { Familiarizar os participantes } \\
\text { com a temática e oferecer } \\
\text { informações práticas de como } \\
\text { fazer um bom uso com as } \\
\text { crianças. }\end{array}$ & $\begin{array}{l}\text { Parte I: Aplicação dos } \\
\text { instrumentos } \\
\text { Parte II: Apresentação } \\
\text { de dados recentes sobre o } \\
\text { fenômeno e particularidades do } \\
\text { uso de mídias digitais na faixa } \\
\text { etária de } 0 \text { a } 3 \text { anos. } \\
\text { Parte } \\
\text { Posicionamento de diferentes } \\
\text { sociedades de Pediatria. } \\
\text { Parte IV: Foram } \\
\text { relatados meios sugeridos pela } \\
\text { literatura científica sobre como } \\
\text { realizar um uso saudável dessas } \\
\text { mídias com as crianças. }\end{array}$ & $\begin{array}{l}\text { Após apresentar brevemente a parte } \\
\text { introdutória, sugere-se enfatizar os } \\
\text { meios relatados pela literatura } \\
\text { científica para um uso saudável na } \\
\text { família. Podem ser disponibilizados } \\
\text { materiais online para os } \\
\text { participantes, como um plano de uso } \\
\text { de mídias digitais a ser preenchido e } \\
\text { implementado pelos membros } \\
\text { familiares. }\end{array}$ \\
\hline
\end{tabular}

Segundo encontro:
$\begin{aligned} & \text { Relação cuidadores } e \\ & \text { as mídias digitais }\end{aligned}$ $\begin{aligned} & \text { Salientar } \\ & \text { importância do vínculo inicial } \\ & \text { cuidadores-criança e a a } \\ & \text { influência que as mídias digitais } \\ & \text { exercem nesse contexto. }\end{aligned}$

Parte I: Aspectos Embora as intervenções
teóricos sobre a importância dos relatadas na literatura científica para vínculos iniciais para redução de tempo de tela em desenvolvimento infantil crianças pequenas sejam saudável. internacionais, é interessante Parte II: Impactos apresentar algumas práticas conhecidos do uso de mídias possíveis e eficazes. Pode ser digitais para a relação disponibilizado para os participantes cuidadores-criança. uma lista de sugestões do que foi Parte III: Relato de encontrado como eficaz, por pesquisa do grupo sobre Rede de exemplo, entregar uma lista de apoio e intervenções para reduzir alternativas para brincadeiras com tempo de tela. os bebês.

\begin{tabular}{lrl}
\hline & Terceiro & \multicolumn{1}{c}{ Informar sobre os } \\
encontro: & Mídias & processos fisiológicos do sono e \\
digitais e os efeitos & $\begin{array}{l}\text { os prejuízos que as mídias } \\
\text { digitais têm no sono, tanto nos } \\
\text { no sono }\end{array}$ & bebês quanto nos adultos. \\
& & Tempo \\
previsto: & 40 minutos
\end{tabular}

Parte I: A $\quad$ Foram utilizados alguns
importância do sono para o recursos, como apresentar o ciclo desenvolvimento infantil circadiano na parte teórica. Além Parte II: Impactos das disso, foram fornecidas orientações mídias digitais no sono $\mathrm{e}$ as sobre a higiene do sono, possíveis hipóteses para esse instrumentalizando-os a pensar impacto. sobre a higiene do sono de sua Parte III: Orientações família. Para profissionais, pode ser para pais e profissionais da saúde entregue um roteiro com sugestões sobre como preservar uma boa de tópicos para avaliação do sono rotina de sono em meio ao em crianças, como rotinas na hora contexto digital. de dormir e atividades durante o dia.

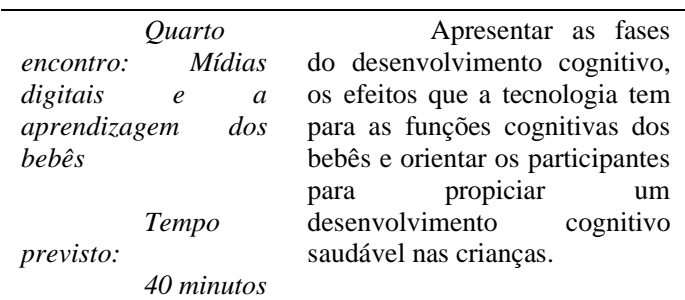

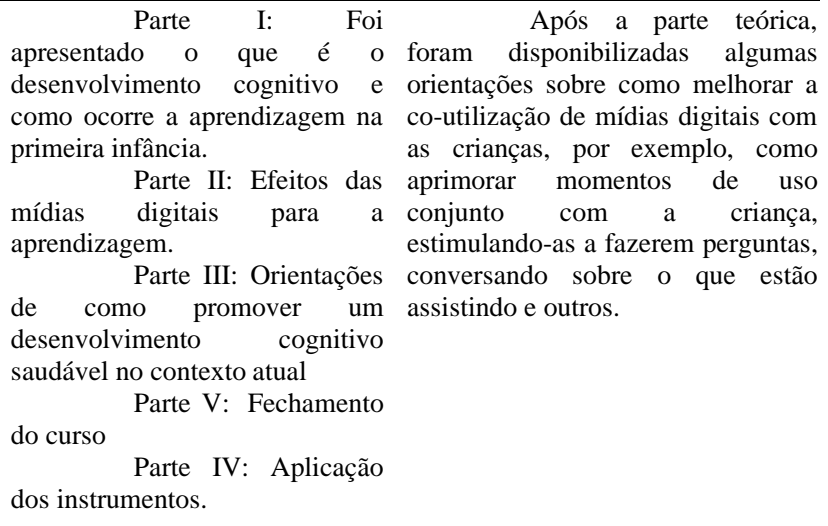

É importante ressaltar que, em todos os encontros, foram exibidos vídeos selecionados da websérie "Bebês \& Tecnologias" e, ao final de cada encontro, havia um momento para discussão e esclarecimento de dúvidas com auxílio da plataforma digital Mentimeter. Essa plataforma possibilita uma apresentação interativa entre participantes e palestrantes, como criação de nuvem de palavras, questionários, perguntas e respostas, enquetes e outros. Enviou-se também, por e-mail, materiais complementares escolhidos 
conforme os assuntos emergentes nas discussões e as demandas dos participantes. Por exemplo, lista de alternativas para brincadeiras com os (as) filhos (as), links para vídeos sobre o assunto e outros.

$\mathrm{Na}$ implementação dessa intervenção, constatou-se a dificuldade de tornar a linguagem científica mais acessível para a população. No entanto, essa é uma tarefa ética crucial para os pesquisadores em Psicologia (Cassepp-Borges, 2009). Embora as pesquisas fossem conhecidas pelas responsáveis pelas palestras, transformar isso em proposições práticas exigiu um esforço adicional. Notou-se que o momento final, em que eram apresentadas propostas e dicas profissionais, geralmente chamava bastante atenção, como por exemplo, o que tinha sido realizado nas intervenções para redução de tempo de tela efetuadas em outros países e relatadas na literatura científica.

Apesar da ampla divulgação e mais de 200 inscrições, outra dificuldade encontrada foi o número de participantes. Esse número reduziu drasticamente ao realizar a confirmação por e-mail e, mais ainda, nos encontros presenciais. Ao longo das palestras, também se observou a diminuição na frequência dos inscritos. $\mathrm{O}$ primeiro encontro contou com 28 participantes, o segundo e terceiro com 22 e o último com 16. Embora o número final ainda esteja próximo ao número planejado inicialmente, é importante considerar em intervenções futuras que seja feito um investimento ainda maior na divulgação.

Mesmo com esses desafios, observou-se grande interesse dos participantes pela temática. Durante os encontros, os relatos de inúmeras situações vividas no cotidiano profissional e familiar que ratificavam as evidências científicas apresentadas pelas ministrantes foi comum. Ao mencionarem suas próprias experiências, foi possível notar uma demanda existente de conhecer alternativas que possam colaborar para um uso mais saudável das telas com os bebês.

À medida que as palestrantes apresentavam algumas possibilidades, os participantes pensavam junto e formulavam outras saídas para as questões que estavam sendo discutidas. Além disso, sempre solicitavam que fossem enviados materiais extras para os ajudarem na empreitada de reduzir ou fazer um melhor uso dessas tecnologias.

Em geral, os participantes eram profissionais de saúde ou educação e cuidadores de uma criança. Essa confluência dos papéis profissional e pessoal propiciou que a intervenção não tivesse um caráter apenas de transferência de conhecimento teóricoprático, mas também reflexivo. Em diversos momentos, eram relatadas reflexões sobre o uso de mídia digital próprio ou da família, especialmente nos encontros que enfatizavam a importância do modo de uso do adulto para o uso da criança. Dessa forma, entende-se que a intervenção cumpriu mais do que a função de transmissão de conhecimento, possibilitando um espaço para ponderações sobre os papéis que as mídias digitais podem desempenhar para o adulto, as crianças e suas famílias.

\section{EVIDÊNCIAS PRELIMINARES DA INTERVENÇÃO}

Para a avaliação da intervenção, foram considerados os seguintes instrumentos: Questionário de conhecimentos sobre o uso de mídias digitais por bebês e Questionário de Satisfação Afetivo-Cognitivo-Comportamental. No primeiro encontro, antes do início das palestras, o Questionário de Conhecimentos foi entregue para preenchimento dos participantes. Em seguida, foi atribuído um código para cada participante e questionário para comparação posterior. Na última palestra, o instrumento foi reaplicado junto com o Questionário de Satisfação. Os dados, então, foram tabulados, digitados e analisados por 
meio do programa estatístico Statistical Package for the Social Sciences (SPSS) versão 24.

Ao todo, a amostra foi composta por 16 participantes que preencheram todos os questionários, sendo que quatorze se identificaram como mulheres e dois como homens. A maior parte eram profissionais de saúde $(n=6)$ ou educação $(n=5)$, seguidos por estudantes da área de educação $(n=2)$, mães $(n=2)$ e um estudante da área da saúde. A intervenção iniciou com 28 participantes e encerrou com 16, registrando uma perda de 43\%. Em geral, uma meta-análise indicou que estudos de intervenção sobre uso de mídias digitais não referem a taxa de perda de participantes (Friedrich et al., 2014). No entanto, o número encontrado neste estudo está em conformidade com outros estudos de intervenção que obtiveram taxas que variaram entre 0\% a 78,2\% (Van der Mispel et al., 2017; Virgili, 2015).

Inicialmente, foram avaliados os instrumentos quantitativamente e, em seguida, a pergunta respondida pelos participantes que constava no Questionário de Conhecimentos. Foi realizada uma análise de estatística descritiva dos dois questionários. A análise dos instrumentos também pretendia avaliar se houve diferença estatisticamente significativa nas pontuações do Questionário de Conhecimentos após a participação na intervenção educativa. Devido à distribuição não normal dos dados, foi aplicado o teste de Wilcoxon para amostras pareadas com objetivo de avaliar a diferença das pontuações do instrumento nos dois momentos pré-intervenção e pósintervenção. O teste de Mann-Whitney também foi utilizado para verificar se houve diferenças no nível de satisfação dos participantes quanto ao número de palestras assistidas (presença nos quatro encontros ou menos).

A análise estatística descritiva mostrou que a média obtida no Questionário de Satisfação Afetivo-Cognitivo-Comportamental em relação à intervenção foi de 52,6 (DP $=2,50$ ) de um total de 55 pontos. Esse valor é considerado alto (Meng et al., 2018), indicando um bom nível de satisfação dos participantes com o curso. Em relação às subescalas, notou-se que a dimensão comportamental foi a que teve maior nível satisfatório $(\mathrm{M}=9,5 ; \mathrm{DP}=0,89)$ de um total de 10 pontos, seguido da dimensão cognitiva $(\mathrm{M}=29,30 ; \mathrm{DP}=1,35)$ de um total de 30 pontos e afetiva $(\mathrm{M}=8,87 ; \mathrm{DP}=$ $0,95)$ de um total de 15 pontos.

No teste de Mann-Whitney, a hipótese nula foi acatada, mostrando que não foram encontradas diferenças estatisticamente significativas no nível de satisfação com relação ao número de palestras assistidas $(\mathrm{U}=44,00 ; p=0.150)$. Outras intervenções educativas com número de encontros próximos a quatro também registraram resultados semelhantes quanto à satisfação (Silva et al., 2018; Torquato et al., 2019). Esse resultado sugere a possibilidade de reduzir o número de encontros para menos de quatro encontros sem prejuízo quanto à satisfação com o curso.

Em relação aos conhecimentos adquiridos, aplicou-se o teste de Wilcoxon para investigar em que medida os níveis de aprendizagem eram equivalentes entre o pré e o pós-teste. De acordo com os dados apresentados na Tabela 2, verificou-se diferença estatisticamente significativa nos escores do Questionário de Conhecimentos aplicados antes e após a participação na intervenção educativa com tamanho de efeito alto $(r=$ 0.67). Esse dado revela que a intervenção é promissora e é encorajadora, na medida que estudos prévios demonstraram que o modelo de intervenção educativa é efetivo não só para transmissão de conhecimentos, como inclusive para redução de uso excessivo de telas por adolescentes e para ampliar as atividades físicas na infância, diminuindo indiretamente o tempo de tela (Gholamian et al., 2019; Harrison et al., 2006). 


\section{Tabela 2}

Comparação entre o conhecimento sobre uso de mídias digitais na primeira infância

\begin{tabular}{lcccc}
\hline Variável & Média & Desvio-padrão & Mediana & valor $p$ \\
\hline $\begin{array}{l}\text { Conhecimentos sobre } \\
\text { digitais na primeira infância }\end{array}$ & & & & \\
Pré-intervenção & 9.06 & 1.56 & 9.5 & \\
Pós-intervenção & 10.5 & 0.81 & 10 & 0.007 \\
\hline \multicolumn{2}{l}{ Nota. Negative Ranks, mean $=3.00 ;$ Positive ranks, mean $=6.30}$. \\
\end{tabular}

Dessa forma, a análise dos dados indica que o estudo-piloto da intervenção educativa sobre uso de mídias digitais na primeira infância parece ter atingido os objetivos iniciais de transmitir conhecimento teórico-prático, ao mesmo tempo em que se constituiu como uma experiência satisfatória para os participantes. Em geral, os cuidadores se apoiam no senso comum ou internet para escolherem ou não usar mídias digitais com as crianças (Covolo et al., 2021). Por isso, intervenções que aprimorem as práticas profissionais sobre esse tópico e tornem informações científicas acessíveis para a população em geral são necessárias.

Esse tema precisa figurar na agenda pública (Straker et al., 2018) e futuras intervenções podem encontrar desafios e possibilidades a partir do presente estudo. Seria interessante se novos estudos investigassem estratégias interventivas eficazes em diferentes regiões do país. Destaca-se que o uso de mídias digitais por bebês, como um fenômeno recente e crescente, faz com que essas intervenções sejam cada vez mais necessárias e aplicadas especificamente no contexto brasileiro.

\section{CONSIDERAÇÕES FINAIS}

No presente estudo, foi possível analisar o processo de construção e implementação do estudo-piloto de uma intervenção educativa sobre uso de mídias digitais por bebês, bem como a avaliação dos conhecimentos adquiridos sobre a temática e a satisfação dos participantes com a intervenção, de forma sistematizada. Destaca-se a avaliação positiva dos participantes acerca da intervenção, com altos índices de satisfação, assim como bons resultados quanto à sua eficácia na aquisição de conhecimentos pelos participantes.

Ainda que tenham sido identificados resultados promissores tanto no modo como foi elaborada a intervenção quanto em seu desfecho, este estudo apresenta algumas limitações. Entende-se que a redução no número de participantes ao longo dos encontros, principalmente no último, foi um aspecto que pode ter influenciado na análise comparativa entre os momentos pré e pós-intervenção. Ademais, destaca-se a ausência de grupo controle e de uma etapa de follow-up para verificar mais precisamente os efeitos da intervenção a médio e longo prazos.

Mesmo assim, este estudo-piloto permitiu identificar informações relevantes a serem consideradas ao elaborar intervenções educativas sobre uso de mídias digitais por bebês no contexto brasileiro, além de adaptar ao Português uma escala de medição de satisfação que se mostrou adequada para avaliar a intervenção. Tanto as dificuldades encontradas, quanto as potencialidades identificadas neste estudo podem elucidar aprimoramentos necessários para futuras intervenções, tais como maior alcance de 
divulgação, melhor adesão dos participantes (seja por redução do número de encontros presenciais, seja por mesclar formato presencial e online) e avaliação dos efeitos da intervenção em médio e longo prazos e seu impacto em variáveis de interesse, por exemplo tempo de tela das crianças. Entretanto, este estudo contribuiu de forma inovadora ao elucidar a criação, avaliação e divulgação de uma intervenção não apresentada na literatura brasileira até então. Por fim, conclui-se que esta se mostrou viável e promissora em seus objetivos e resultados e espera-se que possa contribuir para o avanço desta temática.

\section{REFERÊNCIAS}

American Academy of Pediatrics, Council on Communications and Media. (2016). Media and young minds. Pediatrics, 138(5), 1-8. https://doi.org/10.1542/peds.20162591

American Psychological Association. (2019). Digital media. Thesaurus of psychological index terms.

Borsa, J. C., Damásio, B. F., \& Bandeira, D. R. (2012). Adaptação e validação de instrumentos psicológicos entre culturas: Algumas considerações. Paidéia (Ribeirão Preto), 22(53), 423-432. https://doi.org/10.1590/S0103-863X2012000300014

Brito, R., Francisco, R., Dias, P., \& Chaudron, S. (2017). Family dynamics in digital homes: The role played by parental mediation in young children's digital practices around 14 European Countries. Contemporary Family Therapy, 39(4), 271-280. https://doi.org/10.1007/s10591-017-9431-0

Cassepp-Borges, V. (2009). Devolução de dados por correio eletrônico: Uma alternativa para pesquisas quantitativas. Avaliação Psicológica, 8(1), 149-152. Disponível em: http://pepsic.bvsalud.org/scielo.php?script=sci_abstract\&pid=S1677-

04712009000100015\&lng=pt\&nrm=iso\&tlng=pt

Cheung, C. H. M., Bedford, R., Urabain, I. R. S., Karmiloff-Smith, A., \& Smith, T. J. (2017). Daily touchscreen use in infants and toddlers is associated with reduced sleep and delayed sleep onset. Scientific Reports, $7(1)$. https://doi.org/10.1038/srep46104

Covolo, L., Zaniboni, D., Roncali, J., Mapelli, V., Ceretti, E., \& Gelatti, U. (2021). Parents and mobile devices, from theory to practice: Comparison between perception and attitudes to 0-5 year old children's use. International Journal of Environmental Research and Public Health, 18(7), 3440. https://doi.org/10.3390/ijerph18073440

Coyne, S. M., Radesky, J., Collier, K. M., Gentile, D. A., Linder, J. R., Nathanson, A. I., Rasmussen, E. E., Reich, S. M., \& Rogers, J. (2017). Parenting and digital media. Pediatrics, 140(Supplement 2), S112-S116. https://doi.org/10.1542/peds.2016$1758 \mathrm{~N}$

Domingues-Montanari, S. (2017). Clinical and psychological effects of excessive screen time on children: Effects of screen time on children. Journal of Paediatrics and Child Health, 53(4), 333-338. https://doi.org/10.1111/jpc.13462

Frizzo, G., Bandeira, D., Lewandowski, D., Azevedo, E. C., Mendonça Filho, E., Mallmann, M., Pedrotti, B. G., Pieta, M. A. M., \& Silva, M. (2017). Infants, families, and the use of technologies: A multi-method study for child development. [Unpublished Research Project]. 
Gholamian, B., Shahnazi, H., \& Hassanzadeh, A. (2019). The effect of educational intervention based on BASNEF model for reducing internet addiction among female students: A quasi-experimental study. Italian Journal of Pediatrics, 45(1). https://doi.org/10.1186/s13052-019-0761-4

Harrison, M., Burns, C. F., McGuinness, M., Heslin, J., \& Murphy, N. M. (2006). Influence of a health education intervention on physical activity and screen time in primary school children: 'Switch Off-Get Active'. Journal of Science and Medicine in Sport, 9(5), 388-394. https://doi.org/10.1016/j.jsams.2006.06.012

Kabali, H. K., Irigoyen, M. M., Nunez-Davis, R., Budacki, J. G., Mohanty, S. H., Leister, K. P., \& Bonner, R. L. (2015). Exposure and use of mobile media devices by young children. Pediatrics, 136(6), 1044-1050. https://doi.org/10.1542/peds.2015-2151

Kirkpatrick, J. D., \& Kirkpatrick, W. (2016). Four levels of training evaluation ( $\left.3^{\circ} \mathrm{ed}\right)$. ATD Press.

Lewis, N. L. (2020). Developing a hospital-based postpartum depression education intervention for perinatal nurses. Journal for Nurses in Professional Development, 36(1), 7-11. https://doi.org/10.1097/NND.0000000000000595

Machado, M. C. H. S., Oliveira, J. S., Parada, C. M. G. L., Venâncio, S. I., Tonete, V. L. P., \& Carvalhaes, M. A. B. L. (2010). Avaliação de intervenção educativa sobre aleitamento materno dirigida a agentes comunitários de saúde. Revista Brasileira de Saúde Materno Infantil, 10(4), 459-468. https://doi.org/10.1590/S151938292010000400006

McArthur, B. A., Browne, D., Tough, S., \& Madigan, S. (2020). Trajectories of screen use during early childhood: Predictors and associated behavior and learning outcomes. Computers in Human Behavior, 113, 106501. https://doi.org/10.1016/j.chb.2020.106501

McDaniel, B. T., \& Radesky, J. S. (2018b). Technoference: Longitudinal associations between parent technology use, parenting stress, and child behavior problems. Pediatric Research, 84(2), 210-218. https://doi.org/10.1038/s41390-018-0052-6

Meng, M., Peter, D., Mattner, F., Igel, C., \& Kugler, C. (2018). Development and psychometric pilot-testing of a questionnaire for the evaluation of satisfaction with continuing education in infection control nurses. Nurse Education in Practice, 31, 77-82. https://doi.org/10.1016/j.nepr.2018.05.003

Pedrotti, B. G. (2019). Como prescindir das novas tecnologias no cuidado e na interação com os bebês? [Dissertação de Mestrado], Universidade Federal do Rio Grande do Sul. https://www.lume.ufrgs.br/handle/10183/202058

Radesky, J. S., Silverstein, M., Zuckerman, B., \& Christakis, D. A. (2014). Infant selfregulation and early childhood media exposure. Pediatrics, 133(5), e1172-e1178. https://doi.org/10.1542/peds.2013-2367

Rideout, V., \& Robb, M. B. (2020). The common sense census: Media use by kids age zero to eight, 2020.2 Disponível em: https://www.commonsensemedia.org/sites/default/files/uploads/research/2020_zero _to_eight_census_final_web.pdf

Runfola, C. D., Zucker, N. L., Holle, A. V., Mazzeo, S., Hodges, E. A., Perrin, E. M., Bentley, M. E., Ulman, T. F., Hoffman, E. R., Forsberg, S., Ålgars, M., Zerwas, S., Pisetsky, E. M., Taico, C., Kuhns, R. A., Hamer, R. M., \& Bulik, C. M. (2014). NURTURE: Development and pilot testing of a novel parenting intervention for 
mothers with histories of an eating disorder: Nurture Pilot Testing. International Journal of Eating Disorders, 47(1), 1-12. https://doi.org/10.1002/eat.22178

Schmidt, M. E., Haines, J., O’Brien, A., McDonald, J., Price, S., Sherry, B., \& Taveras, E. M. (2012). Systematic review of effective strategies for reducing screen time among young children. Obesity, 20(7), 1338-1354. https://doi.org/10.1038/oby.2011.348

Silva, F. B. e, Gondim, E. C., Henrique, N. C. P., Fonseca, L. M. M., \& Mello, D. F. de. (2018). Intervenção educativa com mães jovens: Aquisição de saberes sobre cuidados da criança. Acta Paulista de Enfermagem, 31(1), 32-38. https://doi.org/10.1590/1982-0194201800006

Straker, L., Zabatiero, J., Danby, S., Thorpe, K., \& Edwards, S. (2018). Conflicting guidelines on young children's screen time and use of digital technology create policy and practice dilemmas. The Journal of Pediatrics, 202, 300-303. https://doi.org/10.1016/j.jpeds.2018.07.019

Torquato, I. M. B., Collet, N., Forte, F. D. S., França, J. R. F. S., Silva, M. de F. O. C., \& Reichert, A. P. S. (2019). Effectiveness of an intervention with mothers to stimulate children under two years. Revista Latino-Americana de Enfermagem, 27. https://doi.org/10.1590/1518-8345.3176.3216

Van der Mispel, C., Poppe, L., Crombez, G., Verloigne, M., \& De Bourdeaudhuij, I. (2017). A self-regulation-based ehealth intervention to promote a healthy lifestyle: Investigating user and website characteristics related to attrition. Journal of Medical Internet Research, 19(7), e241. https://doi.org/10.2196/jmir.7277

Virgili, M. (2015). Mindfulness-Based interventions reduce psychological distress in working adults: A meta-analysis of intervention studies. Mindfulness, 6(2), 326337. https://doi.org/10.1007/s12671-013-0264-0

Wu, L., Sun, S., He, Y., \& Jiang, B. (2016). The effect of interventions targeting screen time reduction: A systematic review and meta-analysis. Medicine, 95(27), e4029. https://doi.org/10.1097/MD.0000000000004029

Submetido: $15 / 04 / 2021$

Reformulado: 19/10/2021

Aceito: $21 / 10 / 2021$

\section{Sobre as autoras:}

Maíra Lopes Almeida é doutoranda em Psicologia pela Universidade Federal do Rio Grande do Sul (UFRGS).

Laura Canani da Rosa é graduanda em Psicologia pela Universidade Federal do Rio Grande do Sul (UFRGS).

Gabriela Vescovi é doutoranda em Psicologia pela Universidade Federal do Rio Grande do Sul (UFRGS).

Bruna Gabriella Pedrotti é doutoranda em Psicologia pela Universidade Federal do Rio Grande do Sul (UFRGS).

Manoela Yustas Mallmann é doutoranda em Psicologia pela Universidade Federal do 
Rio Grande do Sul (UFRGS).

Giana Bitencourt Frizzo é Doutora em Psicologia pela Universidade Federal do Rio Grande do Sul. Professora do Instituto de Psicologia e do Programa de Pós-graduação em Psicologia da Universidade Federal do Rio Grande do Sul (UFRGS).

Correspondência: maira.lpalmeida@gmail.com 